\title{
Additive Representability of Finite Measurement Structures*
}

\author{
ARKADII SLINKO \\ Department of Mathematics, University of Auckland \\ a.slinko@auckland.ac.nz
}

February 2007; revised March 2008

\begin{abstract}
In this survey we will concentrate on recent developments of Peter Fishburn's ideas in representational theory of finite measurement structures, where he saw a gap in understanding of additive representability of preferences. He made a significant contribution to this theory and formulated a large number of open problems, which will undoubtedly guide future investigators. Only a few of Fishburn's questions have been answered to date. We report on the recent progress in this field, and highlight the remaining unsolved questions. Journal of Economic Literature Classification No.: D81.
\end{abstract}

Keywords: additive conjoint measurement, comparative probability, desirable gamble, multiset.

* I thank participants of The 5th International Simposium on Imprecise Probability: Theories and Applications (ISIPTA 07), Prague, Czech Republic, 16-19 July 2007, and several seminar audiences for comments and suggestions. Financial support from the Economic Design Network (ARC) and the New Zealand Institute of Mathematics and its Applications (NZIMA) is gratefully acknowledged. 


\section{Introduction}

The theory of additive conjoint measurement takes its roots in the papers by Debreu [8] and Luce and Tukey [27]. It is presented in books [34, 13, 23, 26, 42, 35, 33] and excellent surveys, of which Fishburn's survey [17] is the most recent. The goal of the present paper is twofold: we would like to describe some recent developments that took place after Fishburn's survey was published, and to attract attention to several questions posed by Fishburn that remain unanswered.

The main object of this theory is a Cartesian product of finitely many mutually disjoint sets $A_{i}$

$$
A=A_{1} \times A_{2} \times \ldots \times A_{n}
$$

equipped with an order $\preceq$. This product is usually interpreted as the set of alternatives under the consideration of a decision maker, or the set of outcomes that may result from her actions. We may also think that there are $n$ criteria in place and each set $A_{i}$ is identified with the set of levels of the $i$ th criterion. The order represents the decision maker's preference on the set of alternatives.

A decision maker often faces some kind of optimization problem. A solution of this problem would be made feasible if it were possible to find an additive utility representation over criteria of the decision maker's preference order $\preceq$ on $A$. The central theme of the theory of additive conjoint measurement is finding conditions which imply the existence of such a representation. Another important question is about uniqueness of this representation. It appeared that, in many aspects, the most difficult case to study is the case of finite measurement structures, i.e., when $A$ is finite. The main focus of this paper is on this case. In addition to that we restrict ourselves with $\preceq$ being a (strict) linear order, in which case the uniqueness question does not emerge.

Kraft, Pratt and Seidenberg [24] established (see also Scott [37]) that additive utility representation of $\preceq$ is equivalent to a denumerable set of conditions, called cancellation conditions, which is not equivalent to any finite subset of them. However, for a finite Cartesian product of a particular size we need to check only finitely many cancellation conditions for $\preceq$ to establish its additive representability. Fishburn (see, e.g. his motivation of this in $[17,18]$ ) considered that it is extremely important to know the exact number of cancellation conditions needed as a function of the size of the product, or at least a good lower and upper bounds for this number. He saw the absence of such bounds as a serious gap in understanding of additive representability of preferences on finite measurement structures. Fishburn made a significant contribution to this theory and formulated 
a large number of open problems, which have guided and undoubtedly will continue to guide investigators in this area. And although some recent progress has been made, only a few of the great many questions posed by Fishburn have been answered to date.

Let us briefly outline what will be covered in the subsequent sections. In Section 2 we introduce the main types of finite measurement structures considered in the literature to date. They are Cartesian product structure, power set structure, power multiset structure. Section 3 surveys the most general case, the Cartesian product structure. In this case no significant progress has been recently made, and we highlight a number of open questions.

Comparative probability orders, which represent one of the main cases of the power set structure, are surveyed in Section 4. This measurement structure emerges when $A_{i}=\{0,1\}$ for $i=1,2, \ldots, n$, in which case any $n$-tuple of the Cartesian product can be identified with a subset of the set of atoms $[n]=\{1,2, \ldots, n\}$. Here we reformulate the cancellation conditions for comparative probability orders in terms of portfolios of desirable gambles. This framework allows for a better understanding of Fishburn's function $f(n)$, the main object of his investigations in $[14,15]$. We show that $f(n)$ can be interpreted as a measure of rationality of a player required to correctly evaluate any portfolio of gambles with $n$ states of the world. We report on the recent progress in estimation of $f(n)$ and the related function $g(n)$, which was introduced by Conder and Slinko [6]. The reason for introducing this new function is as follows. It is known that for comparative probability orders the absence of arbitrage does not imply additive representation and some cancellation conditions may still be violated. However the absence of arbitrage is a very important condition and $g(n)$ is a complete analogue of $f(n)$ in the situation of no arbitrage.

Fishburn showed by way of a sophisticated combinatorial construction that $f(n) \geq$ $n-1$, which together with the bound $f(n) \leq n+1$ of Kraft-Pratt-Seidenberg [24] gave quite a narrow range for this function. Fishburn conjectured that $f(n)=n-1$. Recently however Conder and Slinko [6] showed that $f(7) \geq 7$ and Marshall [30,31] showed that $f(p) \geq p$ for a large number of prime numbers $p \geq 131$. Conder showed that $f(n) \geq n$ for all $7 \leq n \leq 13$. Fishburn [14, 15] also paid attention to minimal violations of the cancellation conditions which he called irreducible patterns. Here we present a theorem of Matthew Auger [1] which says that there are only finitely many of them.

In Section 5, devoted to power multiset structure, sets are generalised to multisets which allow multiple entry of identical elements. If $A_{i}=\left\{0,1, \ldots, m_{i}\right\}$ and if the $i$ th coordinate of an $n$-tuple from the Cartesian product is $j$, then we may think that the multiset associated with this tuple has $j$ copies of atom $i$. We see great advantages in describing 
this measurement structure in multiset terms, because of the emerging analogies with comparative probability orders. Orders on submultisets of a multiset were first used in the computer science literature by Dershowitz [9] to prove termination of rewrite systems. Sertel and Slinko [39] showed some important applications of multisets in Economics and Political Science. In Economics ranking multisets can be used for ranking income streams and investment projects. In Political Science they can be used for ranking committees or parliaments.

Additive conjoint measurement on subsets of Cartesian products containing rankordered n-tuples was considered by Wakker in [45, 46]. He established that, contrary to what has often been thought, additive conjoint measurement on subsets of Cartesian products has characteristics different from additive conjoint measurement on full Cartesian products.

Fishburn himself did not work with this preference structure but many of his ideas work in this case too. An analogue of de Finetti's axiom here is Independence of Equal Submultisets (IES) introduced in $[39,40]$. The analogues of functions $f(n)$ and $g(n)$ can be introduced and those analogues will have $k$ as an additional parameter, i.e. we obtain functions $f(n, k)$ and $g(n, k)$. It is rather surprising that in this case better progress can be achieved in describing these functions than in the case of comparative probability orders [5]. The function $g(n, k)$ is determined exactly: we have $g(n, k)=n-1$ for $(n, k) \neq(5,2)$ and $g(5,2)=3$. We also have $n \geq f(n, k) \geq g(n, k)$ and we conjecture that $f(n, k)=g(n, k)$.

\section{Types of Finite Measurement Structures}

In this paper we assume that the Cartesian product (1) is finite. Let $m_{i}$ denote the cardinality of $A_{i}$ and in this case the cardinality of the Cartesian product will be $|A|=$ $m_{1} m_{2} \ldots m_{n}$. We interpret $\preceq$ as a nonstrict preference relation on $A$, i.e. $\mathbf{a} \preceq \mathbf{b}$ means $\mathbf{a}$ is not preferred to $\mathbf{b}$. The corresponding strict preference relation $\prec$ and indifference $\sim$ are defined in the usual way.

Sometimes $A_{i}(i=1, \ldots, n)$ are sets without any additional structure. This happens, when elements in each $A_{i}$ belong to the same class but cannot be compared and measured in units of something, e.g., $A_{1}=\{$ apple, banana $\}$ and $A_{2}=\{$ pepsi, coca cola $\}$. Here the Cartesian product consists of pairs

$$
A=\{(\text { apple, pepsi), (apple, coca cola), (banana, pepsi), (banana, coca cola) }\}
$$


We say that we have a Cartesian product structure. The additive utility representation in this case will then take the following form.

Definition 1. A binary relation $\preceq$ on a Cartesian product structure (1) is said to be additively representable if there are $n$ non-negative real-valued functions $u_{i}: A_{i} \rightarrow \mathbb{R}$ such that for all $\mathbf{a}=\left(a_{1}, \ldots, a_{n}\right)$ and $\mathbf{b}=\left(b_{1}, \ldots, b_{n}\right)$ in $A$

$$
\mathbf{a} \preceq \mathbf{b} \Longleftrightarrow \sum_{i=1}^{n} u_{i}\left(a_{i}\right) \leq \sum_{i=1}^{n} u_{i}\left(b_{i}\right) .
$$

An important case emerges when we have $n$ types of goods which are divisible to a certain extent but not infinitely divisible (such as money, cars, houses, etc.). These goods can be measured only in whole units of some quantity which is further indivisible. If the total number of available units of the good of type $i$ is $m_{i}$, then each $A_{i}$ can be identified with the set $\left\{0,1, \ldots, m_{i}\right\}$ which has the structure of the truncated monoid of nonnegative integers $\mathbb{N}_{m_{i}}$. A truncated monoid $\mathbb{N}_{k}=(\{0,1, \ldots, k-1\}, \oplus)$ of positive integers is an algebraic system on the base set $\{0,1, \ldots, k-1\}$, where the addition $\oplus$ is defined as

$$
m \oplus n=\left\{\begin{array}{cc}
m+n & \text { if } m+n<k, \\
\text { undefined } & \text { if } m+n \geq k .
\end{array}\right.
$$

The representability of linear orders on such a Cartesian product must respect the structure on the $A_{i}$ 's, which means that for the $i$ th utility function we must have

$$
u_{i}(k)=k u_{i}(1)
$$

and, in particular, $u_{i}(0)=0$.

When $m_{1}=\ldots=m_{n}=2$, and each $A_{i}$ has the structure of $\mathbb{N}_{2}$, this is the case of goods which are indivisible. A 1 in the $i$ th position of an $n$-tuple $\mathbf{a}=\left(a_{1}, \ldots, a_{n}\right) \in A$ means that the $i$ th good is present in this bundle. The Cartesian product $A$ thus can be identified with the set of all indicator functions on $[n]$ or with the set of all subsets of $[n]$. Then the order $\preceq$ becomes an order on subsets of $[n]$. We call it the power set structure. We will deal only with linear, i.e. antisymmetric orders on subsets, since the general theory has not been developed yet. One obvious necessary condition for additive representability of the power set structure is the famous axiom introduced by de Finetti.

Definition 2. An order $\preceq$ on $2^{[n]}$ is said to satisfy the de Finetti axiom if for any $A, B \in 2^{[n]}$ and any $C \in 2^{[n]}$ such that $C \cap(A \cup B)=\emptyset$

$$
A \preceq B \Longleftrightarrow A \cup C \preceq B \cup C .
$$


If a linear order $\preceq$ satisfies de Finetti's axiom and $\emptyset \prec X$ for any non-empty subset $X \subseteq[n]$, then it is called a comparative probability order. Some significant progress has been recently achieved in understanding of additive representability of comparative probability orders. We report it in Section 4.

A multiset $M$ on a base set $X$ is a collection of elements of $X$, where multiple entries of the same element of $X$ are possible [41]. In general, if $X=\left\{x_{1}, \ldots, x_{k}\right\}$ is a set, then a multiset on $X$ is denoted as $M=\left\{x_{1}^{q_{1}}, x_{2}^{q_{2}}, \ldots, x_{k}^{q_{k}}\right\}$, where $q_{j}$ is the number of occurrences of $x_{j}$ in $M$, respectively. The number $q_{j}$ is normally referred to as the multiplicity of $x_{j}$ in $M$. As some $q_{j}$ may be zero, not all elements of the base set may be present. The number of unique elements of $X$ in $M$ we call the width of $M$ and the sum $\sum_{j=1}^{k} q_{j}$ we call the cardinality of $M$.

When the Cartesian product (1) is such that every $A_{i}$ has a structure of $\mathbb{N}_{m_{i}}$, then $A$ can be identified with all submultisets of the multiset $\left\{1^{m_{i}}, 2^{m_{2}}, \ldots, n^{m_{n}}\right\}$ on $[n]$. In the language of bundles of goods, we have $n$ types of goods, denoted $1,2, \ldots, n$, and exactly $m_{i}$ copies of good $i$ are available. We call it the power multiset structure. The power multiset model has numerous useful interpretations (see e.g., $[40,5]$ ). We report results on the power multiset structure in Section 5.

In some applications not all alternatives of the Cartesian product (1) are actually available for choice. In this case we have to consider orders on a subset of this Cartesian product. Section 6.5.5 of [23] points out the importance of additive conjoint measurement on subsets of Cartesian products. Interest in this topic has increased during the last decade because of new developments in the literature on decision making under risk/uncertainty where conditions like independence are often required to hold only within certain subsets. Sertel and Slinko [39] showed that sometimes from the applications point of view it is necessary to restrict ourselves to the submultisets $\left\{1^{k_{i}}, 2^{k_{2}}, \ldots, n^{k_{n}}\right\}$ of $\left\{1^{m_{i}}, 2^{m_{2}}, \ldots, n^{m_{n}}\right\}$ of fixed cardinality $k$, i.e. those for which $\sum_{i=1}^{n} k_{i}=k$. The set of all submultisets of cardinality $k$ we will denote as $\mathcal{P}_{k}([n])$. They gave several important examples of such applications (see also [5]).

\section{Cartesian Product Structure}

When we deal with sequences of elements of the Cartesian product $A$, we will index them with superscripts, while leaving subscripts to numerate the coordinates of elements of $A$. For example, if $\mathbf{a}^{1}, \ldots, \mathbf{a}^{s}$ is the sequence of elements of $A$, then $a_{9}^{7}$ is the ninth coordinate 
of the seventh vector.

If $\preceq$ is a binary relation on the Cartesian product $A$ and $\mathbf{a} \preceq \mathbf{b}$ is true, then, using the preference elicitation terminology [16], we will say that $\mathbf{a} \preceq \mathbf{b}$ is a valid comparison of the two tuples $\mathbf{a}$ and $\mathbf{b}$.

Definition 3. Let $\preceq$ be a relation on the Cartesian product $A$ and

$$
\mathbf{a}^{1} \preceq \mathbf{b}^{1}, \quad \mathbf{a}^{2} \preceq \mathbf{b}^{2}, \quad \ldots, \quad \mathbf{a}^{q} \preceq \mathbf{b}^{q}
$$

be a sequence of valid comparisons of pairs of elements of $A$ such that $\mathbf{a}^{i} \prec \mathbf{b}^{i}$ for at least one $i$. We say that this sequence has the cancellation property if, for each coordinate $i=1,2, \ldots, n$, the sequence $b_{i}^{1}, b_{i}^{2}, \ldots, b_{i}^{q}$ is a permutation of the sequence $a_{i}^{1}, a_{i}^{2}, \ldots, a_{i}^{q}$.

The number $q$ of comparisons in the sequence (4) will be called its cardinality and the number of unique comparisons in (4) will be called the width of this sequence. Note that this is consistent with the multiset terminology. This is because, if we drop the order of elements in any sequence, it becomes a multiset.

Example 1. The following two sequences of comparisons

$$
\begin{array}{rlrl}
(1,2) & \prec(3,4) & (1,2) & \prec(2,3) \\
(3,4) \prec(1,2) & (2,3) \prec(3,4) \\
& (3,4) \prec(1,2)
\end{array}
$$

the first one, in the left column, of cardinality two, and the second, in the right column, of cardinality three, both have the cancellation property. If all the comparisons of the first sequence are valid for $\preceq$, then $\preceq$ is not antisymmetric, If all the comparisons of the second sequence are valid, then $\preceq$ is not transitive.

From the previous example we get a feeling that having a sequence of valid comparisons with the cancellation property is some kind of a pathology.

Definition 4. We say that a binary relation $\preceq$ on a Cartesian product (1) satisfies the cancellation condition $C_{k}$ if every sequence of comparisons which satisfies the cancellation property has width greater than $k$. We say that a binary relation $\preceq$ satisfies the cancellation condition $C_{k}^{\dagger}$ if every sequence of comparisons which satisfies the cancellation property has cardinality greater than $k$

The following example is taken from Fishburn [17]. 
Example 2. Let $A=\{1,2,3\} \times\{a, b, c\}$. Then the linear order

$$
1 a \prec 1 b \prec 2 a \prec 2 b \prec 3 a \prec 1 c \prec 2 c \prec 3 b \prec 3 c
$$

satisfies $C_{2}$ and $C_{2}^{\dagger}$ but fails both $C_{3}$ and $C_{3}^{\dagger}$ since the sequence of valid comparisons

$$
1 b \prec 2 a, \quad 3 a \prec 1 c, \quad 2 c \prec 3 b
$$

has the cancellation property.

As the width of a multiset is not greater than its cardinality, $C_{k}$ always implies $C_{k}^{\dagger}$. Both $C_{k}$ and $C_{k}^{\dagger}$ group together a large number of conditions but they do it differently. Both are introduced to help us better comprehend the great many cancellation conditions necessary for additive representability.

It is obvious that an additively representable binary relation does not have sequences of valid comparisons that satisfy the cancellation property and, hence, satisfies all cancellation conditions. The converse is also true [23]. The basic rationality assumption for a preference relation on $A$ is called Independence of Equal Subalternatives. It says that for four $n$-tuples $\mathbf{x}, \mathbf{y}, \mathbf{z}, \mathbf{w} \in A$

$$
\mathbf{x} \preceq \mathbf{y} \Longleftrightarrow \mathbf{z} \preceq \mathbf{w}
$$

whenever there exists a proper subset $S \subseteq[n]$ such that $x_{i}=z_{i}$ and $y_{i}=w_{i}$ for all $i \in S$, and $x_{i}=y_{i}$ and $z_{i}=w_{i}$ for all $i \notin S$. We take this terminology from Wakker [44]; Fishburn calls it the first order independence [18]; in [23] this is called coordinate independence. Independence of Equal Subalternatives, being a consequence of $C_{2}$, is not generally sufficient for additive representability. However, as we shall see later, for a limited set of sizes it is true.

Given a relation $\preceq$ on $A$, we may associate the following two numbers with it. Let $f(\preceq)$ be the smallest $k$ such that $\preceq$ violates the cancellation condition $C_{k}$ and $f^{\dagger}(\preceq)$ be the smallest $k$ such that $\preceq$ violates the cancellation condition $C_{k}^{\dagger}$. An obvious relation between these two functions is, of course, $f(\preceq) \leq f^{\dagger}(\preceq)$. However the minimal violation of $C_{k}^{\dagger}$ hypothetically may not have the smallest possible width. Knowing only $f(\preceq)$, we know only half of the story and knowing $f(\preceq)$ and $f^{\dagger}(\preceq)$ gives us the full picture.

Now we will introduce two functions that were of primary interest to Fishburn. We set

$$
f\left(m_{1}, m_{2}, \ldots, m_{n}\right)=\max f(\preceq), \quad f^{\dagger}\left(m_{1}, m_{2}, \ldots, m_{n}\right)=\max f^{\dagger}(\preceq)
$$


where the maximum both times is taken over all binary relations on $A$. In other words, any relation $\preceq$ on $A$, which satisfies cancellation conditions $C_{k}$ with $k \leq f\left(m_{1}, m_{2}, \ldots, m_{n}\right)$ is additively representable and $f\left(m_{1}, m_{2}, \ldots, m_{n}\right)$ is the smallest number with this property. The second function $f^{\dagger}\left(m_{1}, m_{2}, \ldots, m_{n}\right)$ can be similarly characterised. Fishburn concentrated his attention on the first function leaving the second for future research. In this section we will not consider the important case of $\left(m_{1}, m_{2}, \ldots, m_{n}\right)=(2,2, \ldots, 2)$ since we will devote the whole next section to it.

Krantz et al. [23] (see pp. 427-428), who made the initial contribution to this topic, proved that $f\left(2, m_{2}\right)=2$ and that $f(3,3) \geq 3$. Little else was known about these functions until Fishburn's papers $[18,19]$. One of the most significant results of [18] was the general upper bound for $f\left(m_{1}, m_{2}, \ldots, m_{n}\right)$.

Theorem 1 (Fishburn, 1997). $f\left(m_{1}, m_{2}, \ldots, m_{n}\right) \leq \sum_{i=1}^{n} m_{i}-(n-1)$.

As $f\left(2, m_{2}\right)$ is known, the case $n=2$ with $\min \left(m_{1}, m_{2}\right) \geq 3$ naturally attracted much attention $[14,15]$.

Theorem 2 (Fishburn, $1997 \& 2001)$.

1. $f(3,3)=3, f(3,4)=f(4,4)=4$.

2. $f\left(3, m_{2}\right) \geq m_{2}$ for all even $m_{2} \geq 4$, and $f\left(3, m_{2}\right) \geq m_{2}-1$ for all odd $m_{2} \geq 5$.

3. $f\left(m_{1}, m_{2}\right) \geq m_{1}+m_{2}-10$.

4. $f\left(5, m_{2}\right) \geq m_{2}+1$ for all odd $m_{2} \geq 5$.

We note that Theorem 1 gives us $f\left(3, m_{2}\right) \leq m_{2}+2$ so the bounds for $f\left(3, m_{2}\right)$ given by Theorem 2 are rather tight. Apart from obvious questions that these results prompt, Fishburn $[18,19]$ formulated the following interesting ones.

Problem 1. What can be said about $f^{\dagger}\left(m_{1}, m_{2}, \ldots, m_{n}\right)$ ?

Problem 2. We can narrow the class of relations and define the functions $f\left(m_{1}, m_{2}, \ldots, m_{n}\right)$ and $f^{\dagger}\left(m_{1}, m_{2}, \ldots, m_{n}\right)$ for strict linear orders. Will the values of these functions remain the same? Fishburn conjectured that they would (see Conjecture 1 in [18]).

An important paper by Fishburn and Roberts [20] studied the uniqueness question, which we do not survey here due to lack of space. 


\section{Comparative Probability Orders}

As we already noticed, in the case when $m_{1}=\ldots=m_{n}=2$, the Cartesian product $A$ can be identified with the power set of $n$-element set $[n]$. Here we adopt de Finnetti's point of view and consider $[n]$ as the set of the states of the world in which case we can identify comparisons of subsets with gambles. This approach was further developed by Walley and Fine $[47,48,49]$ who believed that there are considerable advantages of basing the theory of comparative probability on desirability of gambles. In our case orders on subsets and desirability of gambles provide two equivalent characterisations but there are some nuances. The shift from preference to desirability is subtle but important. The word "preference" has an optimality flavour while the word "desirability" is more in line with the concept of satisficing introduced by H.A. Simon [43]. The behavioral aspect that can be introduced to comparative probability through the introduction of gambles shed a new light on some old concepts of the theory. In particular, as will be demonstrated below, the functions introduced by Fishburn $[14,15]$ become measures of rationality of personal comparative probability.

\subsection{Discrete Cones}

Let $[n]=\{1,2, \ldots, n\}$ be the set of possible states of the world, one of which will materialise. We suppose that agents can somehow compare probabilities of events. This is their personal probability assessment and it is subjective. If an agent believes that $B$ is more likely to occur than $A$, she should accept the gamble which pays 1 if the state $i \in A \backslash B$ materialises, -1 if the state $i \in B \backslash A$ materialises, and pays nothing in all other cases. On the other hand, if the agent considers this gamble desirable, she must believe that $B$ is more likely to happen than $A$. Thus it is clear that comparative probability assessments of sets and desirability of gambles provide two equivalent languages to discuss orders on subsets. Below we will make this connection formal.

Let $T=\{-1,0,1\}$. Any vector of $T^{n}$ represents a gamble. The gamble which pays $x_{i} \in T$ if the state $i$ materialises will be denoted $\mathbf{x}=\left(x_{1}, \ldots, x_{n}\right) \in T^{n}$. On appearance of a nonzero gamble $\mathbf{x} \in T^{n}$ a participating agent must be ready to accept either $\mathbf{x}$ or $-\mathbf{x}$. The zero gamble $\mathbf{0}$ is neutral (no loss, no profit). Let us agree that it is not desirable.

The following properties will be assumed as basic rationality assumptions that all agents possess:

C1. $\mathbf{e}_{i}=(0, \ldots, 1, \ldots, 0)$ is a desirable gamble for all $1 \leq i \leq n$; 
C2. If $\mathbf{x}$ and $\mathbf{y}$ are two desirable gambles and if $\mathbf{x}+\mathbf{y} \in T^{n}$, then $\mathbf{x}+\mathbf{y}$ is a desirable gamble;

C3. For every nonzero gamble $\mathbf{x} \in T^{n}$, either $\mathbf{x}$ or $-\mathbf{x}$ (but not both) is desirable.

Definition 5. Any subset $\mathcal{C}$ of $T^{n}$ which contains $\mathbf{0}$ and whose nonzero vectors satisfy C1 - C3 is called a discrete cone.

To summarise: the set of desirable gambles for an agent is the set of all nonzero vectors of a certain discrete cone.

For each subset $A \subseteq[n]$ we define the characteristic vector $\chi_{A}$ of this subset by setting $\chi_{A}(i)=1$ if $i \in A$, and $\chi_{A}(i)=0$ if $i \notin A$. For any pair of subsets $A, B \in 2^{[n]}$ we define a gamble:

$$
\chi(A, B)=\chi_{B}-\chi_{A} \in T^{n} .
$$

Given an agent whose set of desirable gambles is a discrete cone $\mathcal{C}$, the agent can compare events as follows:

$$
A \preceq B \Longleftrightarrow \chi(A, B) \in \mathcal{C} .
$$

Due to properties of $\mathcal{C}, \preceq$ is an order (reflexive, complete and transitive relation) on $2^{[n]}$. This order satisfies de Finetti's axiom (3) and hence is a comparative probability. This probability assessment is, of course, specific for this particular agent only.

The study of discrete cones as algebraic objects was initiated by Kumar ${ }^{1}$ in his $\mathrm{PhD}$ thesis [25]. This approach was rediscovered by Fishburn [14] who pioneered their combinatorial study. Further combinatorial properties of discrete cones were studied in $[10,15,16,28,6,30,3]$. In this section we concentrate on combinatorics of rationality assessment.

If $\mathbf{p}=\left(p_{1}, \ldots, p_{n}\right)$ is a probability measure on $[n]$, where $p_{i}$ is the probability of $i$, then we know the probability of every event $A$, by the rule $p(A)=\sum_{i \in A} p_{i}$. We may now define an order $\preceq_{\text {p }}$ on $2^{[n]}$ by

$$
A \preceq_{\mathbf{p}} B \Longleftrightarrow p(A) \leq p(B)
$$

Suppose the probabilities of all events are different. Then $\preceq_{\mathbf{p}}$ is a comparative probability order on $[n]$.

\footnotetext{
${ }^{1}$ I am grateful to Terry Fine for this reference.
} 
Definition 6. Any comparative probability order $\preceq$ on $[n]$ is called additively representable by a measure or simply representable if there exists a probability measure $\mathbf{p}$ on $[n]$ such that $\preceq=\preceq_{\mathbf{p}}$. A comparative probability order $\preceq$ on $[n]$ is said to be almost representable by a measure $\mathbf{p}$ if

$$
A \preceq B \Longrightarrow p(A) \leq p(B) .
$$

In this case we will also say that $\preceq$ is almost representable without specifying the measure $\mathbf{p}$.

If an order $\preceq$ is almost representable but not representable, then at least for one pair of subsets $A$ and $B$ we must have $A \prec B$ and at the same time $p(A)=p(B)$.

\subsection{Portfolios of Acceptable Gambles}

Our way to measure rationality of an agent is to look at how consistent she was in accepting and rejecting various gambles. We need the following concept.

Definition 7. Let $\mathcal{C}$ be a discrete cone. A multiset

$$
P=\left\{\mathbf{x}_{1}^{a_{1}}, \mathbf{x}_{2}^{a_{2}}, \ldots, \mathbf{x}_{m}^{a_{m}}\right\},
$$

where $\mathbf{x}_{i} \in \mathcal{C}$ and $a_{i} \in \mathbb{N}$, is called a portfolio of desirable gambles.

Gambles are like risky securities. You may own different number of shares of the same company. Similarly, a portfolio can contain several identical gambles. If the personal comparative probability of an agent is representable by a measure, then all portfolios of desirable gambles are (in the long run) profitable.

Definition 8. The portfolio $P$ is said to be neutral if

$$
a_{1} \mathbf{x}_{1}+a_{2} \mathbf{x}_{2}+\cdots+a_{m} \mathbf{x}_{m}=\mathbf{0}
$$

The portfolio $P$ is said to be a sure loss if

$$
a_{1} \mathbf{x}_{1}+a_{2} \mathbf{x}_{2}+\cdots+a_{m} \mathbf{x}_{m}=\sum_{i=1}^{n} b_{i} \mathbf{e}_{i}
$$

with $b_{i}<0$ for all $i=1, \ldots, n$.

If a sure-loss portfolio exists, an agent is said to provide an arbitrage. A fully rational agent cannot accept a neutral portfolio and, of course, cannot provide an arbitrage. Here is an example of a comparative probability order that has a neutral portfolio of desirable gambles. 
Example 3. Let $n=5$ and consider the following comparative probability order:

$$
\begin{aligned}
& \emptyset \prec 1 \prec 2 \prec 3 \prec 12 \prec \underline{13 \prec 4} \prec \underline{14 \prec 23} \prec \\
& 5 \prec 123 \prec 24 \prec \underline{34 \prec 15} \prec 124 \prec \underline{25 \prec 134} \ldots
\end{aligned}
$$

(further continuation is unique). The following four desirable gambles

$$
\begin{array}{ll}
\mathbf{x}_{1}=(-1,0,-1,1,0), & \mathbf{x}_{2}=(-1,1,1,-1,0), \\
\mathbf{x}_{3}=(1,0,-1,-1,1), & \mathbf{x}_{4}=(1,-1,1,1,-1)
\end{array}
$$

(they correspond to the underlined comparisons) form a neutral portfolio since $\mathbf{x}_{1}+\mathbf{x}_{2}+$ $\mathbf{x}_{3}+\mathbf{x}_{4}=\mathbf{0}$.

For an example of arbitrage we must have $|\Omega| \geq 6$. Such an example is given in [24]. Conder and Slinko [6] used a computer program to help them find that for $n=6$ there are 5202 such comparative probability orders.

Definition 9. A comparative probability order satisfies cancellation condition $C_{k}$ when no neutral portfolio (7) of desirable gambles of width $k$ exist, and satisfies the cancellation condition $C_{k}^{\dagger}$ when no neutral portfolio (7) of desirable gambles of cardinality $k$ exist.

The criterion of representability given by Kraft et al. [24] can be reformulated as follows.

Theorem 3. Suppose $\preceq$ is the agent's comparative probability order on $2^{[n]}$ and $\mathcal{C}$ be the corresponding discrete cone. Then

1. $\preceq$ is representable iff $\mathcal{C}$ has no neutral portfolios of desirable gambles;

2. $\preceq$ is almost representable iff there is no arbitrage.

\subsection{Fishburn's functions as measures of rationality}

Let $\preceq$ be the agent's comparative probability order. Let $f(\preceq)$ be the smallest width of a neutral portfolio of desirable gambles and $f^{\dagger}(\preceq)$ be the smallest cardinality of a neutral portfolio of desirable gambles, if such portfolios exist. Otherwise set $f(\preceq)=f^{\dagger}(\preceq)=\infty$.

The idea is to measure the agent's rationality by the minimum "size" of the portfolio that she cannot handle properly with accepting a neutral portfolio being the early sign of non-rationality. We have two measures for the size of a portfolio: its width and its cardinality. Each measure gives us a measure of an agent's rationality. They are $f(\preceq)$ 
and $f^{\dagger}(\preceq)$, respectively. The larger these functions are the more rational is the agent. Fishburn defined these functions in terms of cancellation conditions of two types [14]. He and his coauthors used their combinatorial interpretations in terms of multilists [16]. Conder and Slinko [6] used their algebraic reformulation of cancellation conditions in terms of linear dependencies of vectors of discrete cones. However in both cases the real meaning of cancellation conditions is hard to grasp due to the intricacies of those definitions. Portfolios clarify the real meaning of cancellation conditions.

Let $\mathcal{L}_{n}$ be the set of all comparative probability orders on $2^{[n]}$, and let $\mathcal{R}_{n}$ be the set of all almost representable comparative probability orders on $2^{[n]}$. Define

$$
f(n)=\max _{\preceq \in \mathcal{L}_{n}} f(\preceq), \quad f^{\dagger}(n)=\max _{\preceq \in \mathcal{L}_{n}} f^{\dagger}(\preceq),
$$

These two functions were introduced and studied by Fishburn [14, 15]. Also we define

$$
g(n)=\max _{\preceq \in \mathcal{R}_{n}} f(\preceq), \quad g^{\dagger}(n)=\max _{\preceq \in \mathcal{R}_{n}} f^{\dagger}(\preceq) .
$$

These functions were introduced by Conder and Slinko [6]. They are defined similarly to Fishburn's functions, but only for comparative probability orders which do not admit arbitrage. By temporarily setting all orders with arbitrage aside, Conder and Slinko showed that it is possible to achieve some progress and to answer some questions of Fishburn about $f(\preceq)$ and $f^{\dagger}(\preceq)$. The relationships between $f(\preceq)$ and $f^{\dagger}(\preceq)$ and their no arbitrage analogues $g(\preceq)$ and $g^{\dagger}(\preceq)$ are not completely clear. All we can state is that $g(n) \leq f(n)$ and $g^{\dagger}(n) \leq f^{\dagger}(n)$.

Some initial values for these functions are known $[24,14,15,6]$ :

$$
\begin{aligned}
& f(n)=f^{\dagger}(n)=\infty, \quad(n \leq 4), \\
& g(5)=g^{\dagger}(5)=f(5)=f^{\dagger}(5)=4, \\
& g(6)=g^{\dagger}(6)=f(6)=f^{\dagger}(6)=5 .
\end{aligned}
$$

It is also known that $g(n) \leq n[6]$ and we will see later that $g(7)=7$. The following bounds are known for $f(n)$, where the upper bound was established by Kraft et al. [24] and the lower by Fishburn $[14,15]$.

Theorem 4 (Kraft et al., 1959, Fishburn 1997). $n-1 \leq f(n) \leq n+1$.

The upper bound here is a rather trivial fact, the lower bound was obtained by a non-trivial construction. Fishburn $[14,15]$ conjectured that $f(n)=n-1$. However, since $f(n) \geq g(n)$, the first part of the following theorem refutes Fishburn's conjecture. 
Theorem 5 (Conder and Slinko, 2004). $g(7)=7$ and $g^{\dagger}(7) \geq 8$.

This result is based on the following construction theorem.

Theorem 6 (Conder and Slinko, 2004)). Let $X=\left\{\mathbf{x}_{1}, \ldots, \mathbf{x}_{m}\right\} \in T^{n}(m \geq 4)$, such that $\sum_{i=1}^{m} a_{i} \mathbf{x}_{i}=\mathbf{0}$ for some positive integers $a_{i}$, and either no proper subsystem $X^{\prime} \subset X$ is linearly dependent with positive coefficients

or

the sum $\sum_{i=1}^{m} a_{i}$ is minimal possible.

Suppose further that the $m \times n$ matrix $A$ having the vectors $\mathbf{x}_{1}, \ldots, \mathbf{x}_{m}$ as its rows has the property that $A \mathbf{b}=\mathbf{0}$ for some positive integer-valued vector $\mathbf{b}=\left(b_{1}, \ldots, b_{n}\right)$ with $b_{1}>b_{2}>\ldots>b_{n}>0$, and that

$$
\mathbf{b}^{\perp} \cap T^{n}=\left\{ \pm \mathbf{x}_{1}, \ldots, \pm \mathbf{x}_{m}\right\}
$$

Let $\mathbf{p}=\left(b_{1}+\ldots+b_{n}\right)^{-1} \mathbf{b}$ and $C=\left\{\mathbf{x} \in T^{n} \mid(\mathbf{x}, \mathbf{p}) \geq 0\right\}$. Then the discrete cone

$$
C^{\prime}=C \backslash\left\{-\mathbf{x}_{1}, \ldots,-\mathbf{x}_{m}\right\}
$$

corresponds to an almost representable comparative probability order $\preceq$ which almost agrees with $\mathbf{p}$, with either

$$
f(\preceq)=m \quad \text { or } \quad f^{\dagger}(\preceq)=\sum_{i=1}^{m} a_{i},
$$

respectively.

To prove the second part of Theorem 5 one may take the following $7 \times 7$ matrix:

$$
A=\left[\begin{array}{rrrrrrr}
1 & -1 & -1 & 1 & 0 & 1 & -1 \\
1 & 0 & -1 & -1 & 1 & -1 & -1 \\
1 & 0 & -1 & -1 & -1 & 0 & 1 \\
-1 & 1 & -1 & 1 & 1 & 0 & 1 \\
0 & -1 & 1 & 1 & 0 & -1 & 1 \\
0 & -1 & 1 & -1 & 1 & 1 & 1 \\
-1 & 1 & 1 & 0 & -1 & 0 & -1
\end{array}\right]
$$

and let $\mathbf{x}_{1}, \ldots, \mathbf{x}_{7}$ denote its rows. It is easy to check that $\operatorname{rank}(A)=6$,

$$
\mathbf{x}_{1}+\mathbf{x}_{2}+\mathbf{x}_{3}+\mathbf{x}_{4}+\mathbf{x}_{5}+\mathbf{x}_{6}+2 \mathbf{x}_{7}=\mathbf{0}
$$




$$
\operatorname{Span}\left\{\mathbf{x}_{1}, \ldots, \mathbf{x}_{7}\right\} \cap T^{7}=\left\{ \pm \mathbf{x}_{1}, \ldots, \pm \mathbf{x}_{7}\right\}
$$

and $A \mathbf{p}=\mathbf{0}$ for the probability measure

$$
\mathbf{p}=\frac{1}{148}(48,40,27,16,12,10,7)
$$

\subsection{Extremal Cones and Comparative Probability Orders. Mar- shall's Theorem}

In the previous section we saw that discrete cones and comparative probability orders with the property $g(n)=n$ do exist. Since this is the maximal possible value of $g(n)$, Marshall [30] calls such objects extremal. He constructed a great many other extremal comparative probability orders by using some clever algebra and number theory. Before formulating Marshall's theorem we remind the reader that, given a prime $p$, an integer $a$ is called a quadratic residue if there exists a $b$ such that $a=b^{2}(\bmod p)$; otherwise it is called a quadratic non-residue. The Legendre symbol $\left(\frac{a}{p}\right)$ is 0 if $a$ is a multiple of $p, 1$ if $a$ is a quadratic residue $\bmod p$, and -1 if $a$ is a quadratic non-residue.

Theorem 7 (Marshall, 2005). Let $p$ be a prime greater than 131. If

$$
\left(1+\sqrt{\left(\frac{-1}{p}\right) p}\right)^{p}-1=a+b \sqrt{\left(\frac{-1}{p}\right) p},
$$

where $\operatorname{gcd}(a, b)=p$, then there exists an almost representable discrete cone in $T^{p}$ with $g(p)=p$.

The odd primes satisfying the above equation he calls optimus primes. The first few non-optimus primes are

$$
3,23,31,137,191,239,277,359, \ldots
$$

Calculations that he and McCall conducted showed that 1725 of the 1842 primes between 132 and 16000 are optimus primes.

Problem 3. Is the number of optimus primes infinite?

The idea of Marshall's construction is as follows. He uses the construction of Theorem 6 (changing rows into columns) and constructs the matrix needed there by altering the vector of Legendre quadratic residue symbols in the first two co-ordinates as follows:

$$
\mathbf{q}=\left(1,\left(\frac{1}{p}\right)-1,\left(\frac{2}{p}\right), \ldots,\left(\frac{p-1}{p}\right)\right)^{T} .
$$


Then he forms a circulant matrix

$$
Q=\left[\mathbf{q}, S \mathbf{q}, S^{2} \mathbf{q}, \ldots, S^{p-1} \mathbf{q}\right]
$$

from q, where $S$ is the standard matrix of the circular shift operator. Finally he forms $A=Q-E_{11}+E_{1 p}$ which is Marshall's matrix for prime $p$.

Theorem 8 (Conder, 2005). $g(n)=n$ for $7 \leq n \leq 13$.

This result was proved with the help of the MAGMA system [2] and announced in [30]. In the course of achieving it, Conder found that Marshall's matrices work not just for primes $p$ satisfying the conditions given in Theorem 7 , but also for some others, including all primes $p$ in the range $5 \leq p \leq 23$.

A number of questions remain open. The most important ones are:

\section{Problem 4.}

1. Is $f(7)=7$ or is $f(7)=8$ ?

2. What is $g(14)$ ?

3. Is $g(n)=n$ for $n \geq 7$ ?

4. Is it true that $f(n)=g(n)$ ?

5. Does Marshall's construction work for all primes $p \geq 5$ ?

\subsection{Patterns of Minimal Neutral Portfolios}

Definition 10. Let $\preceq$ be a comparative probability order on $2^{[n]}$ and $\mathcal{C}$ be the corresponding discrete cone. Let

$$
P=\left\{\mathbf{x}_{1}^{a_{1}}, \mathbf{x}_{2}^{a_{2}}, \ldots, \mathbf{x}_{n}^{a_{n}}\right\},
$$

be a neutral portfolio of desirable gambles satisfying

1. width $(P)=m$ is minimal possible for a neutral portfolio,

2. for neutral portfolios of desirable gambles of width $m$ the cardinality $\operatorname{card}(P)=$ $\sum_{i=1}^{m} a_{i}$ is minimal. 
In this case we say that $\left(a_{1}, \ldots, a_{m}\right)$ is an irreducible pattern. The set of all irreducible patterns of width $m$ in $2^{[n]}$ is denoted as $\mathcal{A}_{m, n}$. Let us denote

$$
\mathcal{A}_{m}=\bigcup_{n=4}^{\infty} \mathcal{A}_{m, n}
$$

Theorem 9 (Fishburn, 1996).

$$
\begin{aligned}
& \mathcal{A}_{4}=\{(1,1,1,1)\} \\
& \mathcal{A}_{5}=\{(1,1,1,1,1),(1,1,1,1,2)\} .
\end{aligned}
$$

Moreover, $\mathcal{A}_{5,5}=\emptyset$, and $\mathcal{A}_{5}=\mathcal{A}_{5,9}$.

Theorem 10 (Conder-Slinko, 2004).

$$
\begin{aligned}
& \mathcal{A}_{5,6}=\{(1,1,1,1,1)\} \\
& \mathcal{A}_{7,7} \supseteq\{(1,1,1,1,1,1,1),(1,1,1,1,1,1,2)\} .
\end{aligned}
$$

This means that we don't know $\mathcal{A}_{5,7}$ and $\mathcal{A}_{5,8}$. We don't know $\mathcal{A}_{6}$ either. An unpublished recent result in this direction is the following theorem by Auger [1], for which we provide here a short proof.

Theorem 11 (Auger, 2005). For any positive integer $m$ there are only finitely many irreducible patterns of length $\mathrm{m}$.

Proof. Let us consider the set of all vectors of $\mathbb{R}^{m}$ with non-negative integer coordinates. Let us denote it $Z_{m}$. All irreducible patterns from $\mathcal{A}_{m}$ belong to $Z_{m}$. For an arbitrary $\mathbf{a}=\left(a_{1}, \ldots, a_{m}\right) \in Z_{m}$ we denote $h(\mathbf{a})=\sum_{i=1}^{m} a_{i}$. We also define a set

$$
R(\mathbf{a})=\left\{\{I, J\} \mid I, J \subseteq[m], \quad I \cap J=\emptyset, \quad \sum_{i \in I} a_{i}=\sum_{j \in J} a_{j}\right\} .
$$

The set $R(\mathbf{a})$ has a cardinality smaller than the cardinality of the set of all pairs of subsets $\{I, J\}$ with $I \cap J=\emptyset$, which is $\left(3^{m}-1\right) / 2$. Hence $R(\mathbf{a})$ is finite. So it is sufficient to prove that there are only finitely many irreducible patterns a with the same $R(\mathbf{a})$.

Suppose now that we have two irreducible patterns $\mathbf{a}$ and $\mathbf{b}$ with $R(\mathbf{a})=R(\mathbf{b})$. Let $\left\{\mathbf{x}_{1}, \ldots, \mathbf{x}_{m}\right\} \subseteq T^{n}$ such that $\sum_{i=1}^{m} a_{i} \mathbf{x}_{i}=\mathbf{0}$. Then each of the $n$ coordinates of this vector equation will give us an element of $R(\mathbf{a})$ (they will not be necessarily distinct). Hence if another vector $\mathbf{b}=\left(b_{1}, \ldots, b_{m}\right) \in Z_{m}$ will satisfy $R(\mathbf{a})=R(\mathbf{b})$, then $\sum_{i=1}^{m} a_{i} \mathbf{x}_{i}=\mathbf{0}$ will always imply $\sum_{i=1}^{m} b_{i} \mathbf{x}_{i}=\mathbf{0}$ and vice versa. Thus, if $\mathbf{a} \in Z_{m}$ and $\mathbf{b} \in Z_{m}$ are both 
irreducible patterns of $\mathcal{A}_{m}$, then we must have $h(\mathbf{a})=h(\mathbf{b})$. Since there are only finitely many vectors $\mathbf{c}$ in $Z_{m}$ with the given $h(\mathbf{c})$, we see that the set of irreducible patterns a with fixed $R(\mathbf{a})$ is finite and hence $\mathcal{A}_{m}$ is finite.

Problem 5. Let $\mathcal{C}$ be a discrete cone and $P=\left\{\mathbf{x}_{1}^{a_{1}}, \mathbf{x}_{2}^{a_{2}}, \ldots, \mathbf{x}_{m}^{a_{m}}\right\}$ be the neutral portfolio of desirable gambles with the smallest height $\sum_{i=1}^{m} a_{i} . I s\left(a_{1}, \ldots, a_{m}\right)$ an irreducible pattern? Or, in other words, will the width of $P$ also be smallest?

Axioms for unique additive representation of a comparative probability order (which in this case cannot be strict) were given by Fishburn and Roberts [21].

\section{Orders on Submultisets of a Multiset}

In this section we will consider multisets on the base set $[n]$. Every such multiset $M=$ $\left\{1^{m_{i}}, 2^{m_{2}}, \ldots, n^{m_{n}}\right\}$ is uniquely determined by its multiplicity function $\mu:[n] \rightarrow \mathbb{N}$ such that $\mu(i)=m_{i}$. We say that $M_{1}=\left([n], \mu_{1}\right)$ is a submultiset of $M_{2}=\left([n], \mu_{2}\right)$, if $\mu_{1}(i) \leq$ $\mu_{2}(i)$ for all $i \in[n]$, and we denote this by $M_{1} \subseteq M_{2}$. We remind the reader that the set of all submultisets of cardinality $k$ will be denoted as $\mathcal{P}_{k}([n])$.

\subsection{Independence of Equal Submultisets and Additive Repre- sentability}

Definition 11. An order $\preceq$ on $\mathcal{P}_{k}[n]$ is said to be (additively) representable if there exist nonnegative real numbers $u_{1}, \ldots, u_{m}$ (utilities) such that for all $M_{1}=\left([n], \mu_{1}\right)$ and $M_{2}=\left([n], \mu_{2}\right)$ belonging to $\mathcal{P}_{k}[n]$,

$$
M_{1} \preceq M_{2} \Longleftrightarrow \sum_{i=1}^{n} \mu_{1}(i) u_{i} \leq \sum_{i=1}^{n} \mu_{2}(i) u_{i} .
$$

The following basic rationality condition adopted for this situation was suggested by Sertel and Slinko [39, 40], who called it consistency. Here we follow Conder et al. [5] who give a slightly different (but equivalent) definition of this concept, which makes it a close relative to the concept of the Independence of Equal Subalternatives and de Finetti's axiom.

Definition 12. An order $\preceq$ on $\mathcal{P}_{k}([n])$ is said to satisfy the Independence of Equal Submultisets condition (IES) if, for all $1 \leq j \leq k-1$, for every two multisets $U, V$ of cardinality $j$ and for every two multisets $W_{1}, W_{2}$ of cardinality $k-j$,

$$
U \cup W_{1} \preceq V \cup W_{1} \Longleftrightarrow U \cup W_{2} \preceq V \cup W_{2} .
$$


Certainly every additively representable order must satisfy IES. The converse as we will see later is not true. However, it appeared that IES alone implies additive representability on $\mathcal{P}_{k}([3])$ for all $k$. The following theorem was proved first in [39] and later appeared in [40]. We remind the reader of the definition of one of the main number-theoretic functions $\phi$, which is Euler's totient function. For any positive integer $n, \phi(n)$ is the number of positive integers which are smaller than $n$ and relatively prime to $n$. Also, the famous sequence of Farey fractions $\mathbf{F}_{k}$ is the increasing sequence of all fractions in lowest possible terms between 0 and 1 , whose denominators do not exceed $k$. For example, the sequence of Farey fractions $\mathbf{F}_{6}$ will be:

$$
\frac{0}{1}, \frac{1}{6}, \frac{1}{5}, \frac{1}{4}, \frac{1}{3}, \frac{2}{5}, \frac{1}{2}, \frac{3}{5}, \frac{2}{3}, \frac{3}{4}, \frac{4}{5}, \frac{5}{6}, \frac{1}{1}
$$

The standard reference for Farey fractions is [22].

Theorem 12 (Sertel and Slinko, 2002). Any order $\preceq$ on $\mathcal{P}_{k}([3])$ satisfying IES is additively representable. There are $2 \Phi(k)-1$ of them, where $\Phi(k)=\sum_{h=1}^{k} \phi(h)$ and $\phi(h)$ is the Euler totient function, with exactly $\Phi(k)$ orders being strict (antisymmetric). Moreover, if utilities of 1 and 3 are normalized so that $u_{1}=1, u_{3}=0$, then the ith strict order occurs when $u_{2}$ belongs to the ith interval between consecutive Farey fractions in the kth sequence of Farey fractions $\mathbf{F}_{k}$.

Here we will choose a combinatorial way to introduce cancellation conditions similar to Scott's approach [37].

Definition 13. Let $\preceq$ be an order on $\mathcal{P}_{k}[n]$ and let

$$
A_{1} \preceq B_{1}, \quad A_{2} \preceq B_{2}, \quad \ldots, \quad A_{q} \preceq B_{q}
$$

be a sequence of valid set comparisons such that $A_{i} \prec B_{i}$ for at least one $i=1,2, \ldots, q$. We say that this sequence satisfies the cancellation property if the following two multiset unions coincide

$$
A_{1} \cup \ldots \cup A_{q}=B_{1} \cup \ldots \cup B_{q}
$$

Definition 14. We say that an order $\preceq$ on $\mathcal{P}_{k}[n]$ satisfies the $k$ th cancellation condition $C_{k}$ if no sequence of comparisons (12) of width $\leq k$ satisfy the cancellation property and we say that it satisfies the $k$ th cancellation condition $C_{k}^{\dagger}$ if no sequence of comparisons (12) of cardinality $\leq k$ satisfy the cancellation property. 
As in [24, Theorem 2] it is easy to show that for an order $\preceq$ on $\mathcal{P}_{k}([n])$ to be additively representable, it is necessary and sufficient that all cancellation conditions $C_{2}, C_{3}, \ldots, C_{\ell}, \ldots$ are satisfied or alternatively all cancellation conditions $C_{2}^{\dagger}, C_{3}^{\dagger}, \ldots, C_{\ell}^{\dagger}, \ldots$ are satisfied.

Example 4 ([39]). The following linear order on $\mathcal{P}_{2}[4]$

$$
1^{2} \succ 12 \succ \underline{13 \succ 2^{2}} \succ \underline{23 \succ 14} \succ \underline{24 \succ 3^{2}} \succ 34 \succ 4^{2}
$$

satisfies IES but is not representable. It does not satisfy the condition $C_{3}$, since it contains the following comparisons:

$$
\{1,3\} \succ\left\{2^{2}\right\}, \quad\{2,3\} \succ\{1,4\}, \quad\{2,4\} \succ\left\{3^{2}\right\}
$$

Indeed, the union of the multisets on the right and the union of the multisets on the left are both equal to the multiset $\left\{1,2^{2}, 3^{2}, 4\right\}$. Thus $C_{3}$ is violated with $a_{1}=a_{2}=a_{3}=1$, and hence $C_{3}^{\dagger}$ is also violated.

Definition 15. An order $\preceq$ on $\mathcal{P}_{k}[n]$ is said to be almost (additively) representable if there exist nonnegative real numbers $u_{1}, \ldots, u_{m}$, not all of which are equal, such that for all $M_{1}=\left([n], \mu_{1}\right)$ and $M_{2}=\left([n], \mu_{2}\right)$ belonging to $\mathcal{P}_{k}[n]$,

$$
M_{1} \preceq M_{2} \Longrightarrow \sum_{i=1}^{n} \mu_{1}(i) u_{i} \leq \sum_{i=1}^{n} \mu_{2}(i) u_{i} .
$$

If the only way to get $u_{1}, \ldots, u_{n}$ which satisfy $(15)$ is to set $u_{1}=u_{2}=\ldots=u_{n}$, then the order fails to be almost representable. Papers [39, 40] present such an order belonging to $\mathcal{P}_{3}[4]$.

Let $\mathcal{L}_{n, k}$ be the set of all orders on $\mathcal{P}_{k}[n]$ satisfying the IES and $\mathcal{R}_{n, k}$ be the set of all almost representable comparative probability orders on $\mathcal{P}_{k}[n]$ satisfying the IES. As in the case of comparative probability orders we define

$$
f(n, k)=\max _{\preceq \in \mathcal{L}_{n, k}} f(\preceq), \quad f^{\dagger}(n, k)=\max _{\preceq \in \mathcal{L}_{n, k}} f^{\dagger}(\preceq) .
$$

Also we define

$$
g(n, k)=\max _{\preceq \in \mathcal{R}_{n, k}} f(\preceq), \quad g^{\dagger}(n, k)=\max _{\preceq \in \mathcal{R}_{n, k}} f^{\dagger}(\preceq) .
$$

These functions have the same meaning as in the comparative probability orders case. Conder, Marshall and Slinko [5] fully characterized the function $g(n, k)$ as follows: 
Theorem 13. For all $n>3$ and $k \geq 2$,

$$
g(n, k)= \begin{cases}n-2 & \text { if }(n, k)=(5,2) \\ n-1 & \text { otherwise }\end{cases}
$$

This result leaves very little room for the function $f(n, k)$, i.e., $n-1 \leq f(n, k) \leq n$ whenever $(n, k) \neq(5,2)$. Computer-assisted calculations show that $g(n, k)=f(n, k)$ for small values of $n$ and $k$ (namely, for $(n, k)=(4,2),(4,3),(5,2),(5,3),(6,2)$ and $(7,2))$, and so Conder, Marshall, and Slinko conjecture that this is true in general.

Problem 6. Is it true that $f(n, k)=g(n, k)$ for all $n \geq 4$ and $k \geq 1$ ?

Problem 7. What can be said about the relationship between $g^{\dagger}(n, k)$ and $f^{\dagger}(n, k)$ ?

Orders on the infinite set $\mathcal{P}[n]$ of all multisets on $[n]$ satisfying the analogue of the de Finetti axiom (3), where the union is understood as the multiset union and the condition $C \cap(A \cup B)=\emptyset$ is not assumed, were considered by Danilov [7] and Martin [32]. Both independently prove that all orders on $\mathcal{P}[n]$ satisfying this axiom are additively representable. For the set $\mathcal{P}_{\leq k}[n]$ of all multisets on $[n]$ of cardinality $\leq k$, Danilov gives an example of nonrepresentable orders on $\mathcal{P}_{\leq k}[5]$ satisfying the modified de Finetti axiom.

Apart from the aforementioned paper by Danilov, the representability of orders on $\mathcal{P}_{\leq k}[n]$ has largely escaped the attention of researchers. However some interesting things have been observed. For example, it can be easily checked that the linear order on $\mathcal{P}_{\leq 2}[3]$

$$
1^{2} \succ 12 \succ 2^{2} \succ 13 \succ 1 \succ 23 \succ 3^{2} \succ 2 \succ 3 \succ \emptyset
$$

is not representable. Hence the analogue of Theorem 12 is not true.

Problem 8. Develop an additive representation theory for orders on $\mathcal{P}_{\leq k}[n]$.

\section{References}

[1] Auger, M. (2005) Finite Linear Qualitative Probability. Report on The Summer Scholarship Project, Department of Mathematics, The University of Auckland.

[2] Bosma, W., Cannon, J., and Playoust, C. (1997) The Magma Algebra System I: The User Language, J. Symbolic Comput. 24, 235-265. 
[3] Christian, R. and Slinko, A. (2005) Answers to Two Questions of Fishburn on Subset Comparisons in Comparative Probability Orderings, Proceedings of The 4th International Symposium on Imprecise Probabilities and Their Applications (ISIPTA 05), Pittsburg, Pensylvania, 2005, 117-124.

[4] Christian, R., Conder, M. and Slinko, A. (2007) Flippable Pairs and Subset Comparisons in Comparative Probability Orderings, Order, 24(3): 193-213.

[5] Conder, M., Marshall, S., and Slinko, A. (2007) Orders on Multisets and Discrete Cones, Order, 24(4): 277-296.

[6] Conder, M. and Slinko, A. (2004) A counterexample to Fishburn's conjecture on finite linear qualitative probability. Journal of Mathematical Psychology 48, 425-431.

[7] Danilov, V.I. (1987) Aggregation of dichotomic preferences, Mathematical Social Sciences $13,49-58$.

[8] Debreu, G., (1960) Topological methods in cardinal utility theory. In: Arrow, K.J., Karlin, S., and Suppes, P. (Eds.) Mathematical Methods in the Social Sciences. Stanford University Press, Stanford, 16-26.

[9] Dershowitz,N. (1979) Proving termination with multiset orderings. Communications of the ACM 22(8), $465-476$.

[10] Fine, T., and Gill, J., (1976) The enumeration of comparative probability relations. Annals of Probability 4, 667-673.

[11] Fine, T. L. (1973) Theories of probability; an examination of foundations New York, Academic Press.

[12] de Finnetti, B. (1931) Sul significato soggetivo della probabilità, Fundamenta Mathematicae 17, 298-329.

[13] Fishburn, P.C. (1970) Utility Theory for Decision Making, Wlley, New-York.

[14] Fishburn, P.C. (1996) Finite Linear Qualitative Probability, Journal of Mathematical Psychology 40, 64-77.

[15] Fishburn, P.C. (1997) Failure of Cancellation Conditions for Additive Linear Orders, Journal of Combinatorial Designs 5, 353-365. 
[16] Fishburn, P.C., Pekeč A., Reeds, J.A. (2002) Subset Comparisons for Additive Linear Orders, Mathematics of Operations Research 27, 227-243.

[17] Fishburn, P.C. (1999) Preference Structures and Their Numerical Representations, Theoretical Computer Science 217, 359-383.

[18] Fishburn, P.C. (1997) Cancellation Conditions for Multiattribute Preferences on Finite Sets. In: Karwan, M.H., Spronk, J., Wallenius, J. (Eds.) Essays in Decision Making Springer, Berlin, 157-167.

[19] Fishburn, P.C. (2001) Cancellation Conditions for Finite Two-Dimensional Additive Measurement. Journal of Mathematical Psychology 45, 2-26.

[20] Fishburn, P.C., Roberts, F.S. (1988) Unique Finite Conjoint Measurement. Mathematical Social Sciences 16, 107-143.

[21] Fishburn, P.C., Roberts, F.S. (1989) Axioms for Unique Subjective Probability on Finite Sets. Journal of Mathematical Psychology 33, 117-130.

[22] Hardy, G.H., and Wright, E.M. (1960) An Introduction to the Theory of Numbers. Oxford.

[23] Krantz, D.H., Luce, R.D., Suppes, P., and Tversky, A. (1971) Foundations of Measurement (Vol. 1). Academic Press.

[24] Kraft, C.H., Pratt, J.W., and Seidenberg, A. (1959) Intuitive Probability on Finite Sets, Annals of Mathematical Statistics 30, 408-419.

[25] Kumar, A. (1982) Lower Probability on Infinite Spaces and Instability of Stationary Sequences. PhD Thesis. Cornell University.

[26] Luce, R.D., Krantz, D.H., Suppes, P., and Tversky, A. (1988) Foundations of Measurement (Vol. 3). Academic Press.

[27] Luce, R.D. and Tukey, J.W. (1964) Simultaneous conjoint measurement: a new type of fundamental measurement, Journal of Mathematical Psychology 1, 1-27.

[28] Maclagan D. (1999) Boolean Term Orders and the Root System $B_{n}$. Order 15, 279295.

[29] Knuth, D. E. (1998). The Art of Computer Programming - Vol. 2: Seminumerical Algorithms, 3rd edition, Addison Wesley, p.694. 
[30] Marshall, S. (2005) On the Existence of Extremal Cones and Comparative Probability Orderings. Proceedings of The 4th International Symposium on Imprecise Probabilities and Their Applications (ISIPTA 05), Pittsburg, Pennsylvania, 2005, $246-255$.

[31] Marshall, S. (2007) On the Existence of Extremal Cones and Comparative Probability Orderings. Journal of Mathematical Psychology 51(5), 319-324.

[32] Martin,U. (1989) A Geometric Approach to Multiset Ordering, Theoretical Computer Science, 67, 37-54.

[33] Narens, L. (1985) Abstract Measurement Theory, MIT Press, Cambridge MA.

[34] Pfanzagl, J. (1968) Theory of Measurement, Wlley, New-York.

[35] Roberts, F.S. (1979) Measurement theory with applications to decision making, utility, and the social sciences. Reading, MA: Addison-Wesley.

[36] Savage, L.J. (1954) The Foundations of Statistics. New York: Wiley.

[37] Scott, D. (1964) Measurement structures and inequalities, Journal of Mathematical Psychology 1, 233-247.

[38] D. Scott, P. Suppes. (1958) Foundational aspects of theories of measurement. Journal of Symbolic Logic 23, 113-128

[39] Sertel, M., Slinko, A. (2002) Ranking Committees, Words or Multisets. Nota di Laboro 50.2002. Center of Operation Research and Economics. The Fundazione ENI Enrico Mattei, Milan

[40] Sertel, M., Slinko, A. (2007) Ranking Committees, Income Streams or Multisets. Economic Theory 30(2): 265-287.

[41] Stanley, R.P. (1997) Enumerative Combinatorics, Vol.1. Cambridge University Press

[42] Suppes, P., Krantz, D.H., Luce, R.D., and Tversky, A. (1988) Foundations of Measurement (Vol. 2). Academic Press.

[43] Simon, H.A. (1982) Models of Bounded Rationality, Volume 1, Economic Analysis and Public Policy, Cambridge, Mass., MIT Press, 235-441. 
[44] Wakker, P.P. (1989) Additive Representation of Preferences. Kluwer Academic, Dordrecht.

[45] Wakker, P.P. (1991) Additive representations on rank-ordered sets. I. The algebraic approach, Journal of Mathematical Psychology 35, 260-266.

[46] Wakker, P.P. (1993) Additive representations on rank-ordered sets II. The topological approach Journal of Mathematical Economics 22, 1-26.

[47] Walley, P., Fine, T. (1979) Varieties of modal (classificatory) and comparative probability. Synthese 41(3): 321-374

[48] Walley, P. (1991) Statistical Reasoning with Imprecise Probabilities. Chapman and Hall, London.

[49] Walley, P. (1999) Towards a Unified Theory of Imprecise Probability. 1st International Symposium on Imprecise Probabilities and Their Applications, Ghent, Belgium. 